\title{
A novel gridding algorithm to create regional trace gas maps from satellite observations
}

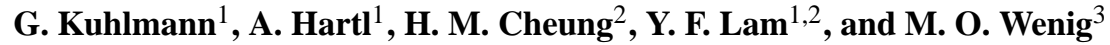 \\ ${ }^{1}$ School of Energy and Environment, City University of Hong Kong, Hong Kong SAR, China \\ ${ }^{2}$ Guy Carpenter Asia-Pacific Climate Impact Centre, School of Energy and Environment, City University of Hong Kong, \\ Hong Kong SAR, China \\ ${ }^{3}$ Meteorologisches Institut, Ludwig-Maximilians-Universität, Munich, Germany
}

Correspondence to: Y. F. Lam (yunflam@cityu.edu.hk)

Received: 26 July 2013 - Published in Atmos. Meas. Tech. Discuss.: 28 August 2013

Revised: 22 November 2013 - Accepted: 12 December 2013 - Published: 10 February 2014

\begin{abstract}
The recent increase in spatial resolution for satellite instruments has made it feasible to study distributions of trace gas column densities on a regional scale. For this application a new gridding algorithm was developed to map measurements from the instrument's frame of reference (level 2) onto a longitude-latitude grid (level 3). The algorithm is designed for the Ozone Monitoring Instrument (OMI) and can easily be employed for similar instruments - for example, the upcoming TROPOspheric Monitoring Instrument (TROPOMI). Trace gas distributions are reconstructed by a continuous parabolic spline surface. The algorithm explicitly considers the spatially varying sensitivity of the sensor resulting from the instrument function. At the swath edge, the inverse problem of computing the spline coefficients is very sensitive to measurement errors and is regularised by a second-order difference matrix. Since this regularisation corresponds to the penalty term for smoothing splines, it similarly attenuates the effect of measurement noise over the entire swath width. Monte Carlo simulations are conducted to study the performance of the algorithm for different distributions of trace gas column densities. The optimal weight of the penalty term is found to be proportional to the measurement uncertainty and the width of the instrument function. A comparison with an established gridding algorithm shows improved performance for small to moderate measurement errors due to better parametrisation of the distribution. The resulting maps are smoother and extreme values are more accurately reconstructed. The performance improvement is further illustrated with high-resolution distributions obtained from a regional chemistry model. The
\end{abstract}

new algorithm is applied to tropospheric $\mathrm{NO}_{2}$ column densities measured by OMI. Examples of regional $\mathrm{NO}_{2}$ maps are shown for densely populated areas in China, Europe and the United States of America. This work demonstrates that the newly developed gridding algorithm improves regional trace gas maps; its application could be very helpful for the study of satellite-derived trace gas distributions.

\section{Introduction}

Satellite retrieved global and regional maps of trace gases are important in many areas of atmospheric research. They provide details about the spatial and temporal distribution of atmospheric components, such as nitrogen dioxide $\left(\mathrm{NO}_{2}\right)$ or sulfur dioxide $\left(\mathrm{SO}_{2}\right)$, which cannot be obtained by groundbased networks or field campaigns alone. Satellite maps have been used to study emissions and transport of trace gases from natural and anthropogenic sources on both a regional and global scale (e.g. Carn et al., 2007). Top-down emission inventories are used to constrain emissions in chemistry transport models (e.g. Martin et al., 2003; Choi et al., 2008). Furthermore, trends and cycles of trace gas concentrations can be identified over regions on different timescales (e.g. Beirle et al., 2003; Richter et al., 2005). Validation studies, comparing space- and ground-based measurements or model simulation, require or can benefit from high-resolution maps to identify the regional distribution of trace gases (e.g. Krotkov et al., 2008; Wenig et al., 2008; Chan et al., 2012). In recent years, the increase in the spatial resolution of satellite 
instruments has made trace gas products more suitable for applications on a regional scale. Examples are the Empa $\mathrm{OMI} \mathrm{NO}_{2}$ product (Boersma et al., 2007; Zhou et al., 2009; Zhou et al., 2010) and the Berkeley High-Resolution (BEHR) product (Russell et al., 2011). These products are retrieved from the Ozone Monitoring Instrument (OMI), which has ground pixel sizes from $13 \times 24 \mathrm{~km}^{2}$ at nadir to $30 \times 160 \mathrm{~km}^{2}$ at the edge of the swath (Levelt et al., 2006).

Vertical column densities of trace gases are typically expressed in the instrument's frame of reference using acrosstrack and along-track position (level 2 product). To visualise and compare the data with other data sets, the level 2 product is projected on a longitude-latitude grid (level 3 product) using a suitable gridding algorithm. On a regional scale, grids may have resolutions between $1 \times 1 \mathrm{~km}^{2}$ and $5 \times 5 \mathrm{~km}^{2}$, which is well within OMI's ground pixel size. At this resolution it is important to consider how the trace gas distribution is reconstructed within the pixel boundaries. A key requirement for a gridding algorithm is the ability to reconstruct shape and extrema of distributions well within the limitation set by the instrument's resolution. It can also be assumed that the distribution is continuous within an orbit of measurements.

Current gridding algorithms typically assume that measurement values are constant within the pixel boundaries. These algorithms produce non-continuous maps and can underestimate maximum values. Within one orbit, consecutive measurements can have overlapping ground pixels due to the instrument slit function. Therefore, Wenig et al. (2008) averaged pixels based on measurement error and pixel size. While their approach conserves the mean value of the measurement, averaging overlapping pixels can further reduce maximum values. A different approach is suggested by Brunner et al. (2007), who tested image reconstruction algorithms used in computed tomography on idealised test data. However, their method targets the reconstruction of monthly or annual maps using a large number of overlapping pixels.

In this paper, we present a novel gridding algorithm which was developed to be more suitable for creating highresolution trace gas maps. The algorithm is designed for OMI, but can easily be employed for similar satellite instruments - for example, the TROPOspheric Monitoring Instrument (TROPOMI), planned for launch in 2014 (Veefkind et al., 2012). TROPOMI is expected to have smaller measurement errors than OMI, due to higher spatial resolution $\left(7 \times 7 \mathrm{~km}^{2}\right.$ at nadir) and better cloud correction.

The new gridding algorithm approximates the trace gas distribution by a continuous differentiable, parabolic spline surface, defined on the lattice of tiled pixels in a single orbit. Our parabolic spline method (PSM) is based on an algorithm developed by Kobza and Mlcak (1994), who construct a spline surface from known mean values on a rectangular lattice. The use of polynomial splines for interpolation is well established (e.g. Lancaster and Salkauskas, 1986; de Boor, 2001). Polynomial approximations which conserve mean values within lattice cells are called histopolations

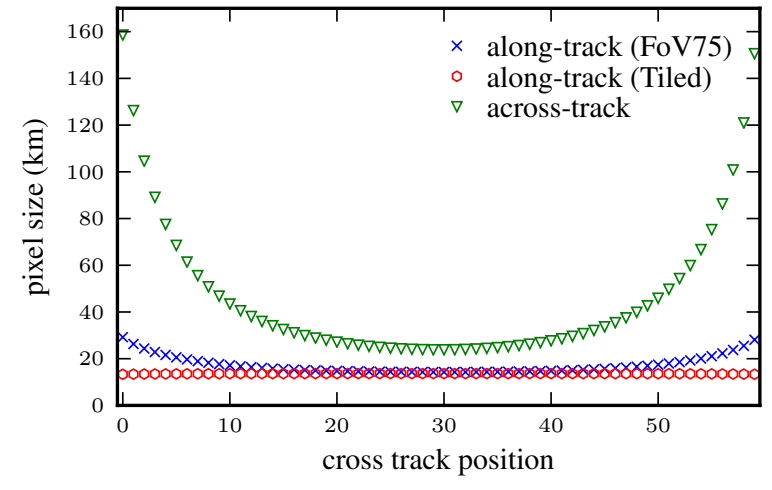

Fig. 1. Along- and across-track ground pixel size for the overlapping (FoV75) and tiled product for the visible channel in the global observation mode. Note that pixels of the tiled product have a constant along-track size of $13 \mathrm{~km}$.

because they are often used for smoothing histograms (Späth, 1995). OMI column densities can be interpreted as weighted averages over the area of a ground pixel which spans several lattice cells. The spatial sensitivity of the sensor over ground is described by the instrument function. PSM derives the mean values within each cell of the lattice considering this instrument function. The inverse problem of computing the mean values from the instrument function is ill-posed in the sense that the retrieved solutions are very sensitive to measurement noise and show a tendency to oscillate around the true mean values if not regularised. A second-order difference matrix is used to regularise the inverse problem. This formulation is similar to the smoothing spline method used to interpolate noisy measurements (de Boor, 2001).

This paper is organised as follows: the measurement principle of OMI is described in Sect. 2.1, and requirements of gridding algorithms are stated in Sect. 2.2. The gridding algorithm described by Wenig et al. (2008) is recapitulated in Sect. 2.3. The parabolic spline method (PSM) is explained in Sect. 2.4, and its performance is evaluated using test scenarios in Sect. 3. The algorithms are applied to produce maps of $\mathrm{OMI} \mathrm{NO} 2$ column densities in Sect. 4. Finally, Sect. 5 contains discussions and conclusions.

\section{Method}

\subsection{Measurement principles}

In this section, we describe the measurement principle of OMI, which is a passive, nadir-viewing imaging spectrometer (Levelt et al., 2006). OMI derives trace gas column densities and aerosol parameters from Earth's reflectance spectra in the near-ultraviolet and visible wavelength range. The reflectance spectrum is scanned along the sunlit part of about 
(a) across-track position: 29

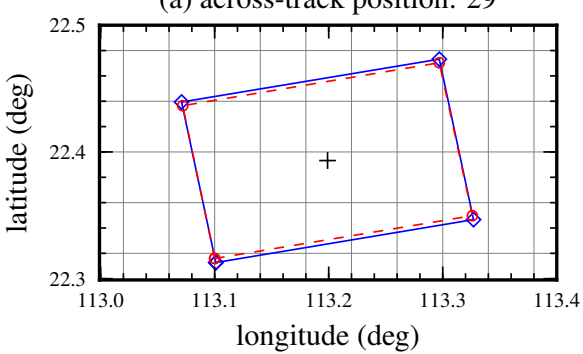

(b) across-track position: 49

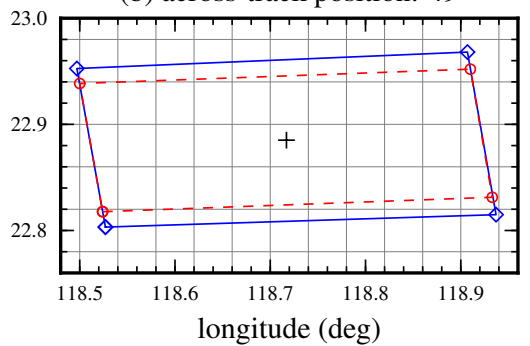

(c) across-track position: 59

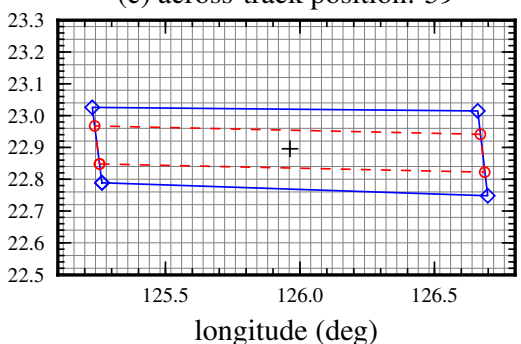

Fig. 2. Pixel shapes for the FoV75 (blue line, diamond) and the tiled (dashed red line, circle) pixel product at across-track positions $i=29$ (nadir), 49 and 59 (swath edge). Pixel centres are marked by a black cross. Note that all pixels have a nearly rectangular shape. A grid with $0.04^{\circ}$ distance is shown for reference. A similar figure is found in Kurosu and Celarier (2010).
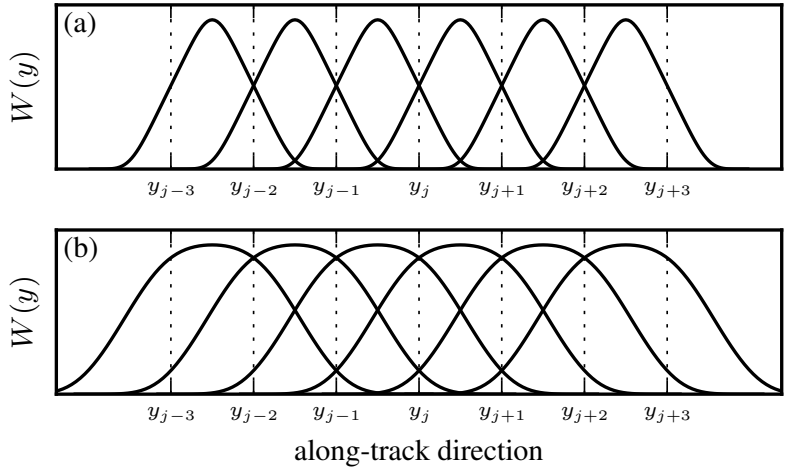

Fig. 3. Normalised instrument functions, as described by Eq. (3), of six consecutive pixels in along-track direction (a) at nadir and (b) at the edge of the swath. The boundaries of the corresponding tiled pixels are shown as dashed, vertical lines.

14.5 sun-synchronous orbits per day, resulting in daily global coverage in a moderate spatial resolution. The scanner applies the push broom principle: a charged-coupled device (CCD) array is used to simultaneously perform $n(=60)$ measurements in across-track direction, resolving a swath width of $2600 \mathrm{~km}$. In along-track direction continuous measurements are averaged every $t(=2) \mathrm{s}$, leading to $m(\approx$ $1600)$ measurement rows in a single orbit. In each orbit, single measurements $\bar{\rho}_{i, j}$ are associated with a two-dimensional $n \times m$ lattice with across-track position $i=0, \ldots, n-1$ and along-track position $j=0, \ldots, m-1$.

A ground pixel is the area at the Earth's surface from which a certain amount of energy is received by the instrument's sensor during a single measurement. Its boundaries are typically described by the four corners of a quadrangle. Geodetic longitude and latitude for the centre of each pixel are included in OMI's level $1 \mathrm{~b}$ data product. The coordinates of the four corners of each ground pixel are provided by the "Ground Pixel Corners Coordinates and Sizes" product (Kurosu and Celarier, 2010), which includes two types of pixel corners:
- Tiled pixel product: adjacent pixels share corners in across- and along-track direction. In along-track direction tiled pixels are always $13 \mathrm{~km}$ long.

- Overlapping product (FoV75): adjacent pixels share corners only in across-track direction, while in alongtrack direction pixels are overlapping, such that the pixel area contains $75 \%$ of the received energy as specified by the instrument function (see below).

The tiled pixel product is useful to create clean maps, where each pixel is recognisable, while the overlapping product better preserves the spatial sensitivity of the instrument and is assumed to be more accurate for derived products, for example emission inventories. The pixel sizes in across- and along-track direction for the two pixel products are shown in Fig. 1. The pixel size of the overlapping pixel increases towards the edge of the swath because of the wider instrument function. At the edge of the swath, overlapping pixels are about twice the length of tiled pixels. Three examples of pixel shapes of both products are shown in Fig. 2. Other products may provide different pixel products: in the Dutch OMI NO 2 (DOMINO) product (Boersma et al., 2011; Dirksen et al., 2011) ground pixels are included as a corner product which is similar to NASA's tiled product.

For each pixel, trace gas column densities $\bar{\rho}_{i, j}$ are retrieved from the measurements with measurement error $\bar{\varepsilon}_{i, j}$. It is convenient to use across-track and along-track directions as $x$ and $y$ axes, respectively. In this coordinate system, the measured column density $\bar{\rho}_{i, j}$ can be expressed in terms of the true distribution $\rho(x, y)$ by

$\bar{\rho}_{i, j}=\int_{A_{i, j}} \rho(x, y) W_{i, j}(x, y) \mathrm{d} A_{i, j}+\bar{\varepsilon}_{i, j}$,

where $A_{i, j}$ is the area over which radiation is received by the sensor. The instrument function $W_{i, j}$ describes the spatial sensitivity of the instrument. For a push broom scanner, we can neglect the $x$ dependency and write the instrument function as

$W_{i, j}(y)=\exp \left(-c y^{4}\right) \odot \operatorname{boxcar}(y)$, 
where $\odot$ is the convolution operator. The first term on the right-hand side is the instrument's slit function and the second term describes the movement of the sensor above ground (Kurosu and Celarier, 2010). The coefficient $c$ is chosen such that the full width at half maximum (FWHM) of the slit function at the Earth's surface corresponds to an instrument's opening angle of $1^{\circ}$. The FWHM depends on the distance between surface and instrument, and hence the width of the instrument function increases towards the edge of the swath (Fig. 3). Since the FWHM changes are small within a pixel, the FWHM can be approximated by the mean value of each pixel. This approximation allows for the implementation of a computationally efficient gridding algorithm using one-dimensional splines. The second factor on the right-hand side of Eq. (2) is the normalised boxcar function, which describes the averaging in along-flight direction in the interval $\hat{y}_{i, j} \pm \frac{1}{2} v t$, where $\hat{y}_{i, j}$ is the pixel centre, $v$ the instrument's speed above ground and $t$ the averaging time. The instrument function is normalised such that the integral is 1 . In the case of OMI, the nadir port moves roughly $13 \mathrm{~km}$ in along-flight direction during each measurement.

\subsection{Gridding algorithm}

A gridding algorithm aims to obtain the best reconstruction of the true distribution of trace gas column densities $\rho(x, y)$ from the measurement values $\bar{\rho}_{i, j}$. The reconstructed distribution $f(x, y)$ is projected on a latitude-longitude grid with indices $u$ and $v$ such that the value at a grid cell $f_{u, v}$ is given by

$$
f_{u, v}=\frac{1}{A_{u, v}} \int_{A_{u, v}} f(x, y) \mathrm{d} A_{u, v},
$$

where $A_{u, v}$ is the area of the grid cell. The resolution of regional maps for trace gas columns vary between 0.01 and $0.05^{\circ}$, corresponding to 1 and $5 \mathrm{~km}$ at $25^{\circ}$ latitude. Since $A_{u, v}$ is much smaller than the ground pixel area $A_{i, j}$ on this scale, $f_{u, v}$ can be approximated by the value of $f(x, y)$ at the centre of the grid cell.

Specific requirements for a gridding algorithm may depend on the particular application. We want our gridding algorithm to have the following properties:

- The weighted average of $f(x, y)$ over the area $A_{i, j}$ should yield the measurement value $\bar{\rho}_{i, j}$ within the error $\bar{\varepsilon}_{i, j}$.

- Since trace gas distributions are continuous, the reconstructed function $f_{u, v}$ should be continuous and have continuous derivatives.

- The shape of plumes and their maximum values should be reconstructed accurately within the measurement error $\bar{\varepsilon}_{i, j}$.
In this paper, the term "lattice" is used to describe the ground pixel in across- and along-track direction (level 2), while the term "grid" is used to describe the longitudelatitude grid of the level 3 product.

\subsection{Constant value method (CVM)}

This section briefly describes a gridding algorithm which assumes constant values of $f(x, y)$ within each pixel's boundaries. CVM is described by Wenig et al. (2008) for $\mathrm{OMI} \mathrm{NO}_{2}$ column densities. CVM is used for quantitative comparison with the parabolic spline method (PSM) presented in this paper.

CVM uses a latitude-longitude grid with a resolution much smaller than the ground pixel size. Each measurement value $\bar{\rho}_{i, j}$ is stored in all grid cells lying within the pixel's boundaries. If pixels overlap, more than one value may be assigned to a given grid cell. In such cases, the weighted average of these values is stored in the cell. Overlap occurs when several orbits are averaged or in consecutive measurements of OMI's FoV75 pixel product. The error in the average is minimised if the weight $\omega_{i, j}$ is proportional to the inverse of the squared measurement uncertainty $\delta_{i, j}^{2}$. Therefore, pixel weights are computed by

$\omega_{i, j}=\frac{1}{A_{i, j} \delta_{\varepsilon_{i, j}}^{2}}$

with pixel size $A_{i, j}$ (Wenig et al., 2008). The disadvantage of CVM is that the reconstructed distribution can have discontinuities at the pixel boundaries. Furthermore, maximum values can be underestimated (see Fig. 6) because the mean value of a trace gas distribution is usually lower than the largest value within a ground pixel. This effect is stronger for pixels with a wider instrument function because the area of the pixel is larger and thus CVM averages pixels with larger overlap.

\subsection{Parabolic spline method (PSM)}

In order to derive more realistic distributions, we developed a new gridding algorithm. The trace gas distribution is described by a parabolic spline surface on the rectangular lattice formed by OMI's tiled pixel product. Parabolic splines are used because they are the simplest, continuous functions which can preserve mean values. In this section, we first consider one-dimensional splines in across- and along-track direction, which are used to calculate two-dimensional surfaces afterwards.

\subsubsection{Across-track direction}

In across-track direction, we have $n$ pixels, whose boundaries $\left(x_{i}, x_{i+1}\right)$ are an increasing sequence of $n+1$ knots:

$\left\{x_{i}\right\}_{i=0}^{n}=\left\{x_{i}: x_{0}<x_{1}<\ldots<x_{n-1}<x_{n}\right\}$, 
with $x_{0}$ and $x_{n}$ being the left and right edge of the swath. A parabolic spline $f(x)$ on the interval $\left[x_{0}, x_{n}\right]$ is written as the sum of piecewise-defined, parabolic polynomials $f_{i}$, which are defined by

$f_{i}(x)= \begin{cases}\sum_{k=0}^{2} a_{i}^{(k)} \Phi_{k}(x) & \text { for } x \in\left[x_{i}, x_{i+1}\right], \\ 0 & \text { otherwise. }\end{cases}$

Each polynomial is described by three coefficients $a_{i}^{(k)}$ and a basis of parabolic polynomials $\Phi_{k}$. It is common (e.g. Kobza and Mlcak, 1994) to introduce the dimensionless variable $s$ given by

$s=\frac{x-x_{i}}{x_{i+1}-x_{i}}=\frac{x-x_{i}}{h_{i}}$

and to formulate $f_{i}(s)$ on the unit interval using the coefficients $d_{i}, p_{i}$ and $p_{i+1}$ defined by

$$
\begin{aligned}
f_{i}(s=0) & =p_{i}, \\
f_{i}(s=1) & =p_{i+1}, \\
\int_{0}^{1} f_{i}(s) \mathrm{d} s & =d_{i} .
\end{aligned}
$$

With these definitions, the polynomial $f_{i}(s)$ is

$$
f_{i}(s)=p_{i} \Phi_{p_{0}}(s)+p_{i+1} \Phi_{p_{1}}(s)+d_{i} \Phi_{d}(s),
$$

with base functions

$$
\begin{gathered}
\Phi_{p_{0}}(s)=1-4 s+3 s^{2} \\
\Phi_{p_{1}}(s)=-2 s+3 s^{2} \\
\Phi_{d}(s)=6 s-6 s^{2} .
\end{gathered}
$$

The advantage of this formulation is that the coefficients have an obvious physical meaning as values of the distribution, and thus their consistency can be validated easily. The values at the knots are given by the $n+1$ coefficients $p_{i}$ and the mean values over the intervals are given by the $n$ coefficients $d_{i}$. Furthermore, it follows directly from Eqs. (8) and (9) that the spline is continuous.

In across-track direction, we assume that the mean values $d_{i}$ are given by the measurement values $\bar{\rho}_{i}$. Therefore, we only need to determine the knot values $p_{i}$, which is typically done by requiring continuity of the first derivative of the spline $f^{\prime}$ at each knot $x_{i}$. This is written as

$$
\frac{1}{h_{i-1}} f_{i-1}^{\prime}(1)=\frac{1}{h_{i}} f_{i}^{\prime}(0) \text {, }
$$

leading to the following equation:

$$
\frac{p_{i-1}}{h_{i-1}}+\left(\frac{2}{h_{i-1}}+\frac{2}{h_{i}}\right) p_{i}+\frac{p_{i+1}}{h_{i}}=3\left(\frac{d_{i-1}}{h_{i-1}}+\frac{d_{i}}{h_{i}}\right) .
$$

On an interval with boundaries $x_{0}$ and $x_{n}$, two additional boundary conditions are required to close the system. Common choices are the natural boundary conditions:

$$
\begin{aligned}
f_{0}^{\prime}\left(x_{0}\right)=0 \quad & \Rightarrow \quad-4 p_{0}-2 p_{1}=-6 d_{0} \\
f_{n-1}^{\prime}\left(x_{n}\right)=0 \quad & \Rightarrow \quad 2 p_{n-1}+4 p_{n}=6 d_{n-1} .
\end{aligned}
$$

To compute the $n+1$ coefficients $p_{i}$, the $n-1$ continuity conditions (Eq. 14) and the two boundary conditions (Eq. 15) are written as matrix equation

$\mathbf{C} \boldsymbol{x}=\mathbf{0}$.

The parameter vector $\boldsymbol{x} \in \mathbb{R}^{2 n+1}$ is defined as

$\boldsymbol{x}=\left[\begin{array}{llllllll}p_{0} & d_{0} & p_{1} & d_{1} & \ldots & p_{n-1} & d_{n-1} & p_{n}\end{array}\right]^{T}$

and $\mathbf{C} \in \mathbb{R}^{(n+1) \times(2 n+1)}$ is a band matrix of the form

$\mathbf{C}=\left[\begin{array}{ccccc}2 \alpha_{0} & -3 \alpha_{0} & \alpha_{0} & & \\ \alpha_{0} & -3 \alpha_{0} & 2\left(\alpha_{0}+\alpha_{1}\right) & -3 \alpha_{1} & \alpha_{1} \\ \ldots & \cdots & & \\ \alpha_{n-2} & -3 \alpha_{n-2} & 2\left(\alpha_{n-2}+\alpha_{n-1}\right) & -3 \alpha_{n-1} & \alpha_{n-1} \\ & \alpha_{n-1} & -3 \alpha_{n-1} & 2 \alpha_{n-1}\end{array}\right]$

with $\alpha_{i}=1 / h_{i}$. Equation (16) can be used as a constraint (see Sect. 2.4.2), or, if the mean values $d_{i}$ are known, it can be used to compute the $n+1$ coefficients $p_{i}$. In the latter case, matrix $\mathbf{C}$ can be simplified to a tridiagonal matrix. The parameter vector $\boldsymbol{x}$ is computed by inverting this simplified matrix using a decomposition algorithm for a tridiagonal matrix.

\subsubsection{Along-track direction}

In along-track direction the same spline, Eq. (11), is used on the $m+1$ knots of the tiled pixel

$\left\{y_{j}\right\}_{j=0}^{m}=\left\{y_{j}: y_{0}<y_{1}<\ldots<y_{m-1}<y_{m}\right\}$.

The $m$ measurement values $\bar{\rho}_{j}$ are obtained by the convolution of the true distribution $\rho(y)$ with the instrument function $W_{j}(y)$ - see Eq. (2) - which now takes the form

$\bar{\rho}_{j}=\int_{-\infty}^{+\infty} \rho(y) W_{j}(y) \mathrm{d} y+\bar{\varepsilon}_{j}$

with measurement error $\bar{\varepsilon}_{j}$. Likewise, the weighted mean value $\bar{f}_{j}$ can be derived from the spline function $f(y)$ as

$\bar{f}_{j}=\sum_{l=j-r}^{j+r}\left[\int_{y_{l}}^{y_{l+1}} f_{l}(y) W_{j}(y) \mathrm{d} y\right]$.

The integer $r$ is chosen such that the integral over $W_{j}(y)$ contains $99 \%$ of the total area under the curve. In this case 


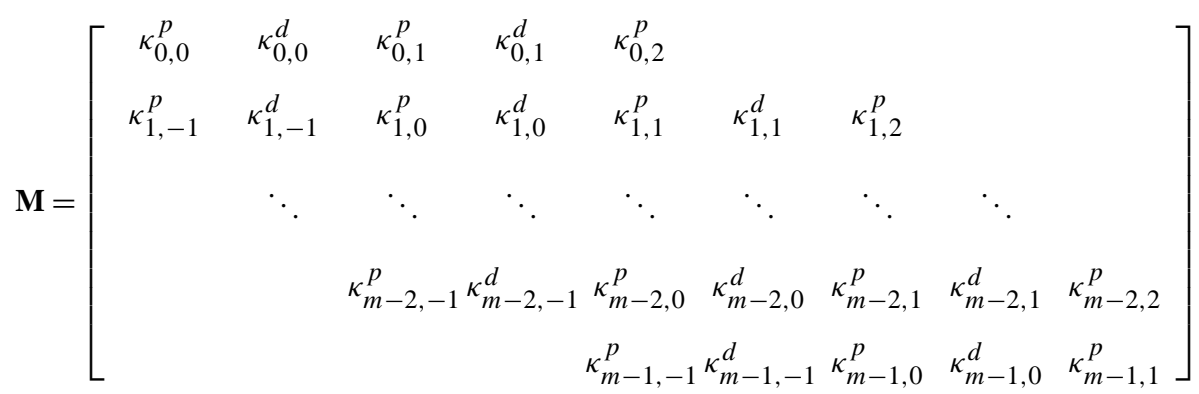

Fig. 4. The structure of matrix $\mathbf{M}$ for an instrument function $W_{j}(y)$ computed over three pixels $(r=1)$.

$r=1$ (i.e. integral over three pixels) at nadir and $r=2$ (integral over five pixels) at the edge of the swath. Examples of instrument functions are shown in Fig. 3. Substituting Eq. (11) in Eq. (21) shows that the equation is linear in the spline coefficients $p_{j}$ and $d_{j}$. The factors of each coefficient are computed by numerical integration of Eq. (21) for a given instrument function.

To compute the $m$ coefficients $d_{j}$, the $m$ measurement equations (Eq. 21) are written as matrix equation

$\mathbf{M} x=y$

with parameter vector $\boldsymbol{x} \in \mathbb{R}^{2 m+1}$ and data vector $\boldsymbol{y} \in \mathbb{R}^{m}$ :

$\boldsymbol{y}=\left[\begin{array}{lllll}\rho_{0} & \rho_{1} & \ldots & \rho_{m-2} & \rho_{m-1}\end{array}\right]^{T}$.

Equation (22) relates the data vector $\boldsymbol{y}$ to the weighted mean values $\bar{f}_{j}$ using matrix $\mathbf{M}$. Each row of the band matrix $\mathbf{M}$ has entries of the factors of the spline coefficients as calculated by Eq. (21). If the instrument function $W_{j}(y)$ is computed over three pixels $(r=1)$, the matrix $\mathbf{M}$ is as in Fig. 4 , where $\kappa_{j, k}^{d}$ is the factor of coefficient $d_{j+k}$ calculated by

$\kappa_{j, k}^{d}=\int_{y_{j+k}}^{y_{j+k+1}} \Phi_{d}(y) W_{j}(y) \mathrm{d} y$

with base function $\Phi_{d}(y)$ given by Eq. (12). The factor $\kappa_{j, k}^{p}$ of coefficient $p_{j+k}$ is computed by

$\kappa_{j, k}^{p}=\int_{y_{j+k-1}}^{y_{j+k}} \Phi_{p_{1}}(y) W_{j}(y) \mathrm{d} y+\int_{y_{j+k}}^{y_{j+k+1}} \Phi_{p_{0}}(y) W_{j}(y) \mathrm{d} y$.

The factors depend on the knots $y_{j}$, the distance between satellite and ground pixel and the exposure time (see Eq. (2)). They can be computed by numerical integration.

\subsubsection{Quadratic programming problem}

The parameter vector $\boldsymbol{x}$ can be computed from Eqs. (16) and (22). The inverse problem is ill-posed, i.e. very sensitive to measurement noise, if the FWHM of the instrument function is about twice the length of the pixel size. The problematic solutions have mean value coefficients $d_{j}$ which fluctuate around their true values; that is, the values are alternately too small and too large. This issue worsens with increasing measurement uncertainty. To prevent over- and undershooting, the inverse problem of retrieving $\boldsymbol{x}$ is written as a leastsquare problem, which minimises a quadratic objective function $\varphi(\boldsymbol{x})$ with an additional penalty term to regularise the inversion:

$\varphi(\boldsymbol{x})=(\mathbf{M} \boldsymbol{x}-\boldsymbol{y})^{T} \mathbf{S}_{\varepsilon}^{-1}(\mathbf{M} \boldsymbol{x}-\boldsymbol{y})+\gamma\left(\mathbf{L}_{2} \boldsymbol{x}\right)^{T} \mathbf{B}^{-1}\left(\mathbf{L}_{2} \boldsymbol{x}\right)$,

$\mathbf{S}_{\varepsilon}$ being the covariance matrix, which here is assumed to be diagonal. The second term (on the right-hand side) is the penalty term, which is weighted by the smoothing parameter $\gamma$. The matrix $\mathbf{L}_{2} \in \mathbb{R}^{(m-2) \times(2 m+1)}$ is the second-order difference matrix, which is used for the mean values $d_{j}$ in $\boldsymbol{x}$ only:

$\mathbf{L}_{2}=\frac{1}{3}\left[\begin{array}{cccccccccccc}0 & 1 & 0 & -2 & 0 & 1 & 0 & & & & \\ & & 0 & 1 & 0 & -2 & 0 & 1 & 0 & & \\ & & & \ddots & \ddots & \ddots & \ddots & \ddots & \ddots & \ddots & \\ & & & & 0 & 1 & 0 & -2 & 0 & 1 & 0\end{array}\right]$.

The penalty term computes the sum of the squared deviations of $d_{j}$ from the local mean of three consecutive coefficients and thus prevents strong local oscillation. The diagonal matrix $\mathbf{B}$ is used to cancel the units of the penalty term and to reduce the dependency of the optimal smoothing on the measurement uncertainty $\delta_{\varepsilon}$ (see Sect. 3.2). We set the entries of $\mathbf{B}$ as

$b_{j j}=\rho_{\mathrm{est}} \delta_{\varepsilon_{j}}$,

where $\rho_{\text {est }}$ is an estimate of the maximum value of the distribution.

Adding the continuity constraint given by Eq. (16), the computation of the spline can be written as a linearly constrained quadratic programming problem:

minimise $\varphi(x)=\frac{1}{2} \boldsymbol{x}^{T} \mathbf{H}_{\gamma} \boldsymbol{x}+\boldsymbol{g}^{T} \boldsymbol{x}+k$

subject to $\mathbf{C} \boldsymbol{x}=\mathbf{0}$ 


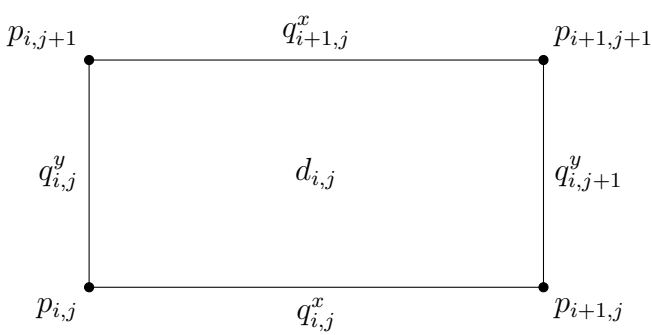

Fig. 5. The nine coefficients of a biparabolic polynomial defined by Kobza and Mlcak (1994).

with

$$
\begin{aligned}
\mathbf{H}_{\gamma} & =2 \mathbf{M}^{T} \mathbf{S}_{\varepsilon}^{-1} \mathbf{M}+2 \gamma \mathbf{L}_{2}^{T} \mathbf{B}^{-1} \mathbf{L}_{2} \\
\boldsymbol{g} & =-2 \boldsymbol{y}^{T} \mathbf{S}_{\varepsilon}^{-1} \mathbf{M} \\
k & =\boldsymbol{y}^{T} \mathbf{S}_{\varepsilon}^{-1} \boldsymbol{y}
\end{aligned}
$$

where the Hessian matrix $\mathbf{H}_{\gamma} \in \mathbb{R}^{(2 m+1) \times(2 m+1)}$ depends on the smoothing parameter $\gamma$. Equation (29) is rewritten using the method of Lagrangian multipliers as

$$
\left[\begin{array}{rr}
\mathbf{H}_{\gamma} & \mathbf{C}^{T} \\
\mathbf{C} & \mathbf{0}
\end{array}\right]\left[\begin{array}{l}
\boldsymbol{x} \\
\lambda
\end{array}\right]=\left[\begin{array}{c}
-\boldsymbol{g} \\
\mathbf{0}
\end{array}\right]
$$

where $\lambda$ is the vector of Lagrangian multipliers (Nocedal and Wright, 2006). The parameter vector is calculated by inverting the sparse matrix of Eq. (31) using a standard solver for sparse linear systems. Other strategies to solve quadratic programming problems can be found in Nocedal and Wright (2006).

\subsubsection{Two-dimensional algorithm}

In this section, we show how a continuous two-dimensional surface spline can be constructed using the one-dimensional splines just described. The algorithm is based on an algorithm described by Kobza and Mlcak (1994), who computed a surface spline for known mean values $d_{i, j}$. The use of one-dimensional splines allows for computation of the coefficients by inverting several small matrices. In contrast, computing all coefficients simultaneously requires the inversion of one very large matrix. The latter case has two major disadvantages: (1) the computational complexity of matrix algebra increases more than linearly with the size of the matrix and (2) a larger matrix is more prone to floating-point inaccuracy.

The two-dimensional spline surface is defined on the nearly rectangular lattice formed by the tiled pixel product:

$$
\left\{\left(x_{i}, y_{j}\right): x_{0}<x_{1}<\ldots<x_{n}, y_{0}<y_{1}<\ldots<y_{m}\right\} .
$$

Following Kobza and Mlcak (1994), each piecewise defined polynomial is written as

$$
\begin{aligned}
f_{i, j}(s, t) & =\Psi_{00} p_{i, j}+\Psi_{10} p_{i+1, j}+\Psi_{01} p_{i, j+1} \\
& +\Psi_{11} p_{i+1, j+1}+\Psi_{x 0} q_{i, j}^{x}+\Psi_{x 1} q_{i, j+1}^{x} \\
& +\Psi_{y 0} q_{i, j}^{y}+\Psi_{y 1} q_{i+1, j}^{y}+\Psi_{d} d_{i, j}
\end{aligned}
$$

using nine coefficients (see also Fig. 5) defined by

$$
\begin{aligned}
p_{i, j} & =f_{i, j}\left(x_{i}, y_{j}\right) \\
q_{i, j}^{x} & =\int_{x_{i}}^{x_{i+1}} f_{i, j}\left(x, y_{j}\right) \mathrm{d} x \\
q_{i, j}^{y} & =\int_{y_{j}}^{y_{j+1}} f_{i, j}\left(x_{i}, y\right) \mathrm{d} y \\
d_{i, j} & =\int_{x_{i}}^{x_{i+1}} \int_{y_{j}}^{y_{j+1}} f_{i, j}(x, y) \mathrm{d} y \mathrm{~d} x
\end{aligned}
$$

and the basis functions

$$
\begin{aligned}
& \Psi_{00}(s, t)=(1-s)(1-t)(1-3 s-3 t+9 s t) \\
& \Psi_{10}(s, t)=s(1-t)(-2+3 s+6 t-9 s t) \\
& \Psi_{01}(s, t)=t(1-s)(-2+6 s+3 t-9 s t) \\
& \Psi_{11}(s, t)=s t(4-6 s-6 t+9 s t) \\
& \Psi_{x 0}(s, t)=6 s(1-s)(1-t)(1-3 t) \\
& \Psi_{x 1}(s, t)=6 s t(1-s)(3 t-2) \\
& \Psi_{y 0}(s, t)=6 t(1-s)(1-t)(1-3 s) \\
& \Psi_{y 1}(s, t)=6 s t(1-t)(3 s-2) \\
& \Psi_{d 0}(s, t)=36 s t(1-s)(1-t) .
\end{aligned}
$$

Kobza and Mlcak (1994) calculate the nine coefficients in three steps by solving several one-dimensional splines (Eq. 16). The first step of their algorithm requires the knowledge of the mean values $d_{i, j}$. In our application, these mean values are unknown. Therefore, we modify the first step to compute the mean values from the measurement values $\bar{\rho}_{i, j}$ as described in Sect. 2.4.3. The coefficients are computed following these three steps:

Step 1. We start with the measurement values $\bar{\rho}_{i, j}$ and estimated standard deviations $\delta_{\varepsilon_{i, j}}$ to solve Eq. (31) for each along-track column $i$. An appropriate smoothing parameter $\gamma$ has to be chosen in this step (see Sect. 3):

$$
\begin{aligned}
\text { for } i & =0, \ldots, n-1: \\
\boldsymbol{y} & =\left[\bar{\rho}_{i, 0}, \ldots, \bar{\rho}_{i, m-1}\right] \\
\boldsymbol{e} & =\left[\delta_{i, 0}, \ldots, \delta_{i, m-1}\right] \\
& \\
\mathbf{M} & =\text { (Fig. 4) } \\
\mathbf{S}_{\varepsilon} & =\text { (Eq. 26) } \\
\mathbf{B} & =\text { (Eq. 28) }
\end{aligned}
$$




$$
\gamma=(\text { Sect. 3) }
$$

Solve Eq. (31) to obtain $\boldsymbol{x}=\left[q_{i, 0}^{x}, d_{i, 0}, \ldots, d_{i, m-1}, q_{i, m}^{x}\right]$.

Thus, this step computes the mean values $d_{i, j}$ and the line integrals along the boundaries of the lattice cells $q_{i, j}^{x}$.

Step 2. Since the mean values $d_{i, j}$ are known, they can be used to compute the coefficients $q_{i, j}^{y}$ for each row in across-track direction:

$$
\text { for } \begin{aligned}
& j=0, \ldots, m-1: \\
& \text { Solve Eq. }(16) \\
& - \text { with mean values: }\left[d_{0, j}, \ldots, d_{m-1, j}\right] \\
& - \text { to obtain }\left[q_{0, j}^{y}, \ldots, q_{n, j}^{y}\right] .
\end{aligned}
$$

Step 3. Finally, the line integral $q_{i, j}^{x}$ can be used as mean values to compute the coefficients $p_{i, j}$ for each row in across-track direction:

$$
\begin{aligned}
& \text { for } j=0, \ldots, m: \\
& \quad \text { Solve Eq. }(16) \\
& \quad \text { - with mean values }\left[q_{0, j}^{x}, \ldots, q_{m-1, j}^{x}\right] \\
& \quad \text { to obtain }\left[p_{0, j}, \ldots, p_{n, j}\right] .
\end{aligned}
$$

Now all coefficients are known and the values of the distribution $f_{u, v}$ can be calculated at each grid cell. The borders of each pixel are calculated to obtain all grid cells which lie within the pixel. Next, the relative coordinates $s$ and $t$ are computed for the centre of each grid cell to calculate the value of the distribution $f_{u, v}=f_{i, j}(s, t)$ by Eq. (34).

\subsubsection{Handling of missing values}

In observational data, trace gas values may be missing for some pixels due to unavailable measurement values or retrieval errors. In along-track direction, missing values could be omitted in the minimisation problem Eq. (26) and thus the spline coefficients at these pixels would be determined by the penalty term and neighbouring pixels. However, the reconstruction of the surface spline should compute missing values using the measurement in both along- and in across-track direction. Therefore, we estimate all missing values from available measurements using bilinear interpolation. The estimated values are included with low weight in Eq. (26) using an (estimated) measurement uncertainty $\delta_{\varepsilon}$ equal to the estimated maximum value $\rho_{\text {est }}$.

By this method the entire spline surface of an orbit can be determined. However, only the parts of the spline surface are projected on the grid for which measurement values are available. Optionally, interpolated values can be kept in the gridded data product to fill small data gaps.

\section{Performance evaluation}

In this section, test scenarios are used to study the performance of PSM and to determine the optimal smoothing parameter $\gamma$. Another important aspect addressed in this section is an analysis of the impact of measurement noise on the algorithm performances using Monte Carlo simulations. Finally, the performance of PSM is compared with CVM under different test scenarios.

\subsection{Reconstruction errors}

Four types of errors may occur when reconstructing a distribution. First we have to consider that the parametrisation of the surface using constant values or parabolic polynomials may not be able to provide an adequate representation of the true distribution (parametrisation error). Furthermore, errors may occur due to the inaccurate description of the measurement process and its numeric implementation (forward model error). Another error type is the perturbation error, which results from the propagation of the measurement error through the model. The oscillation of the mean values caused by measurement noise is a perturbation error. Finally, smoothing errors can occur in PSM if the penalty term is given too much weight. We define the best possible representation as the one without (or with negligible) perturbation and smoothing errors.

We use the root-mean-square deviation (RMSD) between true and reconstructed distribution as a measure for the overall error of the gridded result:

$l_{2}=\sqrt{\frac{1}{N} \sum_{u, v}\left(\rho_{u, v}-f_{u, v}\right)^{2}}$,

where $\rho_{u, v}$ and $f_{u, v}$ are the true and reconstructed distributions, respectively, $u$ and $v$ are the grid indices and $N$ is the number of grid cells.

Furthermore, the absolute difference between maximum and minimum value is used to identify how well maxima are reconstructed by the algorithms:

$l_{\max }=\sqrt{\left(\rho_{u_{\max }, v_{\max }}-f_{u_{\max }, v_{\max }}\right)^{2}}$,

where $u_{\max }$ and $v_{\max }$ are the grid coordinates of the maximum in the true distribution.

These error measures depend on the grid resolution. The continuous distribution created by PSM will only be of advantage if the grid resolution is smaller than the ground pixel size. Here we use a grid resolution 10 times smaller than the pixel size, which is $1.3 \mathrm{~km}$ for OMI.

\subsection{Performance for test scenarios}

In this section the performances of PSM and CVM are compared for simple test scenarios and the characteristics of the different types of errors are discussed. Since PSM is based 
(a) 1-D across-track direction

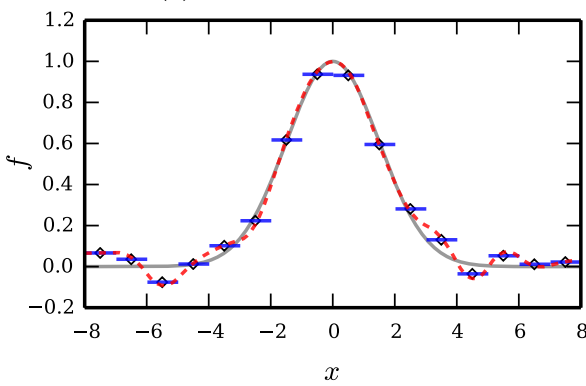

(c) 1-D along (near the edge, $i=55$ ) for $\gamma=5$

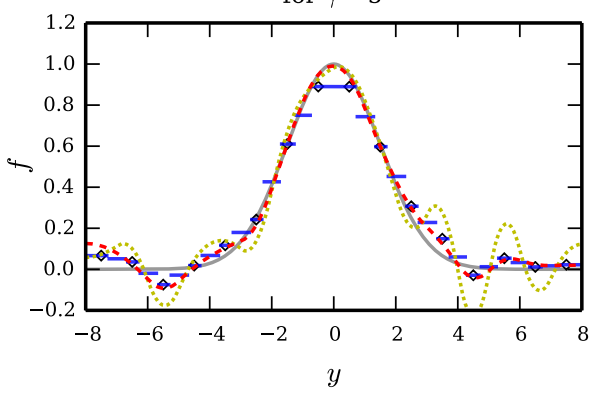

(b) 1-D along (nadir, $i=29$ )

for $\gamma=1$

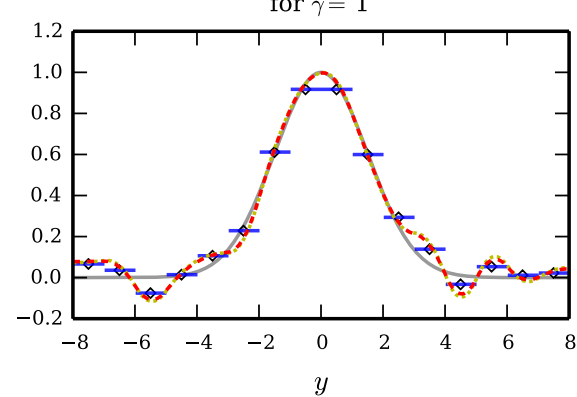

(d) 1-D along (swath edge, $i=59$ )

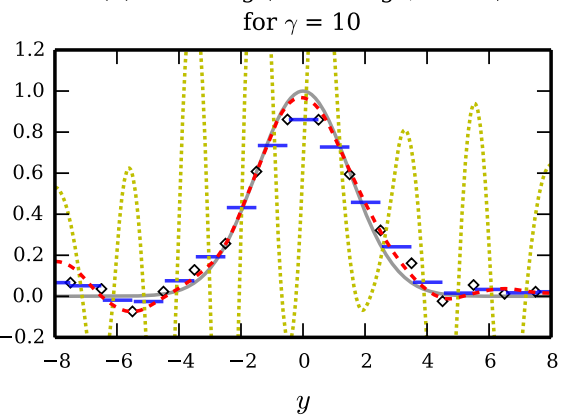

- true $\diamond \diamond$ "measurements" $\rho_{j} \quad-\operatorname{CVM} \quad \ldots \quad \operatorname{PSM}(\gamma=0) \quad--\quad \operatorname{PSM}(\gamma$ optimal $)$

Fig. 6. Reconstruction of a one-dimensional plume $(\sigma=1.5 h)$ using CVM and PSM in (a) across-track direction and in along-track direction for instrument functions (b) at nadir, (c) near the swath edge and (d) at the edge of the swath. The reconstruction using PSM is shown with optimal and without $(\gamma=0)$ regularisation. The measurement values $\rho_{j}$ (with $\delta_{\varepsilon}=5 \%$ ) are drawn at the centre of each pixel.
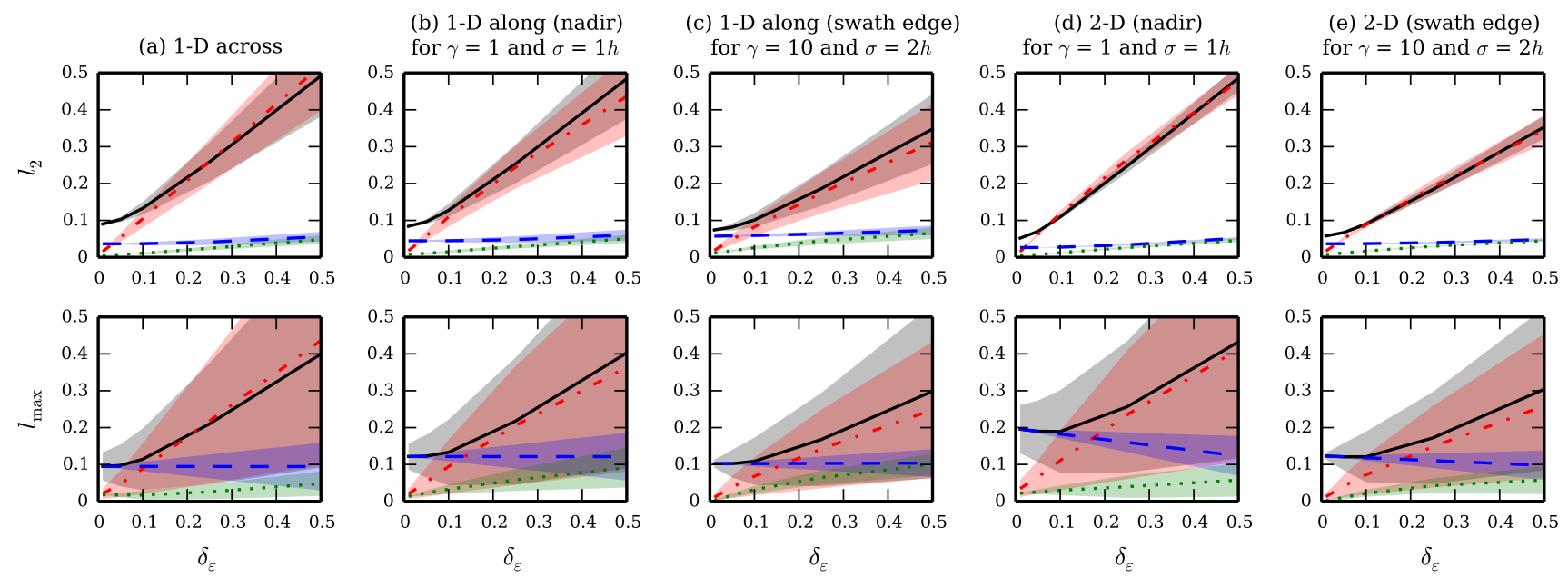

$\operatorname{CVM}(k=1)$

$\operatorname{PSM}(k=1)$

- $-\operatorname{CVM}(k=100)$

$\operatorname{PSM}(k=100)$

Fig. 7. Overall error $\left(l_{2}\right)$ and error of the maximum $\left(l_{\max }\right)$ of CVM and PSM for the reconstruction of a plume using different measurement noise $\delta_{\varepsilon}$. Lines indicate sample averages and the shaded areas are the standard deviations. The errors are shown for $k$ times averaged reconstructions. The errors for the one-dimensional reconstructions are shown in (a) across-track and (b, c) along-track direction at nadir and the edge of the swath, respectively. The errors for the two-dimensional algorithms are shown (d) at nadir and (e) at the edge of the swath. 

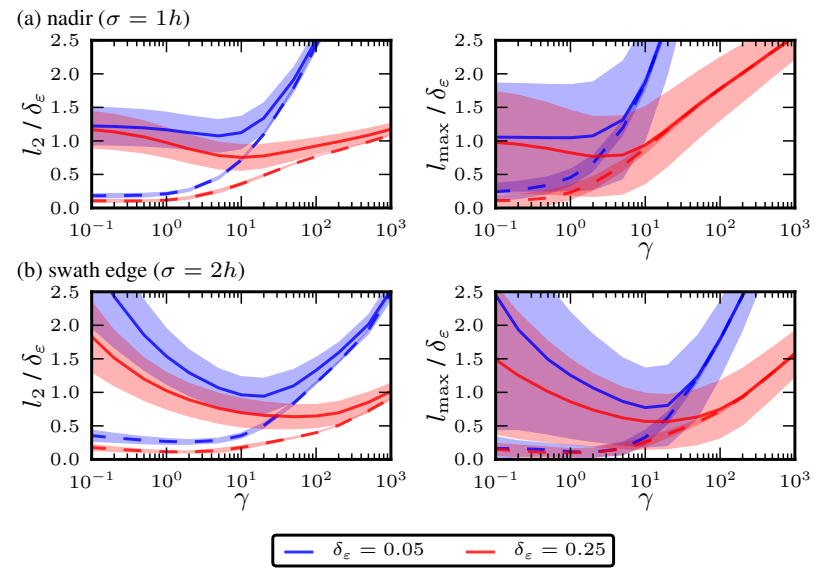

Fig. 8. Dependency of the errors $l_{2}$ and $l_{\max }$ on the smoothing parameter $\gamma$ for a single plume (one-dimensional case) and two levels of measurement noise. Solid lines for the sample mean of a single reconstruction and dashed lines for the average of 100 reconstructions. The shaded areas are the standard deviations.

on separate one-dimensional reconstructions, we first evaluate the performance of this algorithm for the two dimensions separately and then for two-dimensional reconstructions.

\subsubsection{Monte Carlo simulation}

Our test scenarios consist of single plumes and a constant background distribution. Trace gas plumes are created by localised emissions over cities or industrial areas. Constant values can be expected in remote regions without major point emissions. The plumes are modelled by Gaussian functions with standard deviations, $\sigma$, between $1 h$ and $2 h$ (with pixel size $h$ ), which are typical sizes of plumes found in OMI's $\mathrm{NO}_{2}$ product. The maximum value of the plumes is one.

The distributions are discretized on a one- or twodimensional grid with $x$ and $y$ being across- and along-track direction, respectively. Simulated measurement values are obtained by convolving the test distribution with the instrument function, Eq. (1), and selecting discrete values on an equidistant lattice of 11 pixels in across-track and 11 pixels in along-track direction. The position of the lattice on the test distribution is shifted between samples to mimic different instrument positions over ground. In along-track direction, instrument functions with different widths (FWHM) are tested for positions between nadir and the edge of the swath.

The measurement errors are treated as random Gaussian noise with standard deviations from 1 to $75 \%$ of the plumes' maximum values. Our main objective is to reconstruct tropospheric $\mathrm{NO}_{2}$ column densities, whose uncertainties are mainly below $25 \%$ under good viewing conditions (cloud cover $\leq 50 \%$ ). This assumption is based on typical errors, which are $0.1 \times 10^{16} \mathrm{~cm}^{-2}$ for clear sky and about $0.5 \times 10^{16} \mathrm{~cm}^{-2}$ for cloud fractions of $50 \%$ (Bucsela et al.,
2013). Maximum $\mathrm{NO}_{2}$ column densities are typically about 2 to $10 \times 10^{16} \mathrm{~cm}^{-2}$ over polluted regions.

The performance is studied for single reconstructions and for averages of 100 reconstructions with different errors. Averaging measurements is common practice to reduce noise and to study, for example, annual distribution. For the different test scenarios a range of smoothing parameters $\gamma$ is analysed to determine optimal smoothing.

\subsubsection{Across-track direction}

In across-track direction, the spline $f_{u}(x)$ is obtained by computing its parameters using Eq. (16). If a constant distribution is reconstructed, the $l_{2}$ errors are growing linearly with increasing $\delta_{\varepsilon}$ for CVM and PSM (not shown). The reason is that forward model and parametrisation errors are small and the $l_{2}$ error is dominated by the perturbation error, which originates from the propagation of the measurement errors $\delta_{\varepsilon}$. In this scenario PSM is more sensitive to $\delta_{\varepsilon}$ than CVM and thus $l_{2}$ errors of PSM are slightly larger. Since the measurement uncertainty $\delta_{\varepsilon}$ is modelled as random noise, averaging $k$ measurements will reduce the $l_{2}$ error by the inverse square root of $k$. Consequently, $l_{2}$ is reduced by 10 for $k=100$ for both algorithms.

Figure 6a illustrates the reconstruction of a plume $(\sigma=$ 1.5h) using PSM and CVM for small errors. It can be seen that the reconstruction with PSM is more accurate than with CVM. The dependency of $l_{2}$ and $l_{\max }$ on the uncertainty $\delta_{\varepsilon}$ is shown in Fig. 7a. As for the constant distribution, the error grows with increasing uncertainty. However, in this test scenario the best possible representation is limited by the parametrisation error, as can be seen for small errors $\left(\delta_{\varepsilon}<20 \%\right)$. If CVM is used, the error of the best possible reconstruction is considerably larger than for PSM. For this reason, PSM performs better than CVM for small and moderate errors. On the other hand, a larger sensitivity of PSM to $\delta_{\varepsilon}$ causes larger errors for $\delta_{\varepsilon}$ greater than $25 \%$ if PSM is used instead of CVM. The error of $k$ averaged measurements decreases with the inverse square root of $k$, but converges towards a best possible representation (see Fig. 7a for $k=100)$. The $l_{2}$ error of CVM for $k=100$ averages is smaller than the best possible representation of a single measurement. The reason is that the movement of the lattice between samples improves the parametrisation of the distribution. This error reduction cannot be found for the $l_{\max }$ error. Overall, the performance of PSM is better than CVM if the measurement error is small or is reduced by averaging.

\subsubsection{Along-track direction}

In along-track direction, the coefficients of spline $f_{v}(y)$ are computed by solving Eq. (31) for a given smoothing parameter $\gamma$. The penalty term is scaled using the inverse of matrix $\mathbf{B}$, which is proportional to the measurement uncertainty $\delta_{\varepsilon}$ and $\rho_{\text {est }}$ Eq. (28). $\rho_{\text {est }}$ is set to 1 . Using this scaling, $l_{2}$ and 
(a) CMAQ

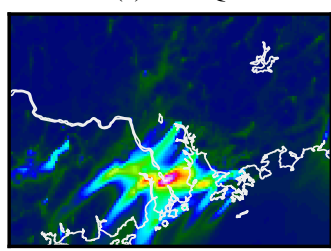

(b) CVM (nadir)

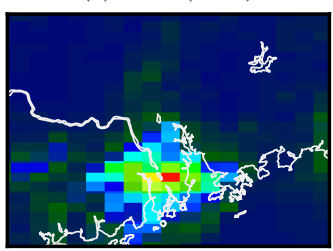

(c) PSM (nadir)

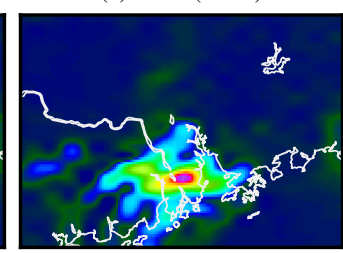

(d) CVM (swath edge)

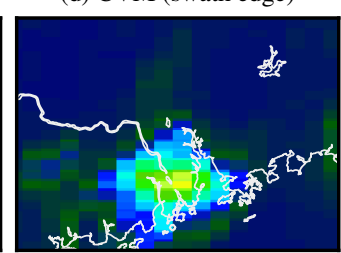

(e) PSM (swath edge)

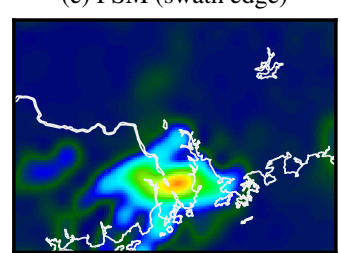

Fig. 9. (a) $\mathrm{NO}_{2}$ column densities simulated by CMAQ on 8 October 2012, 14:00 LT, and (b)-(e) reconstructions of distribution with CVM and PSM for a measurement uncertainty $\delta_{\varepsilon}$ of 0.05 .

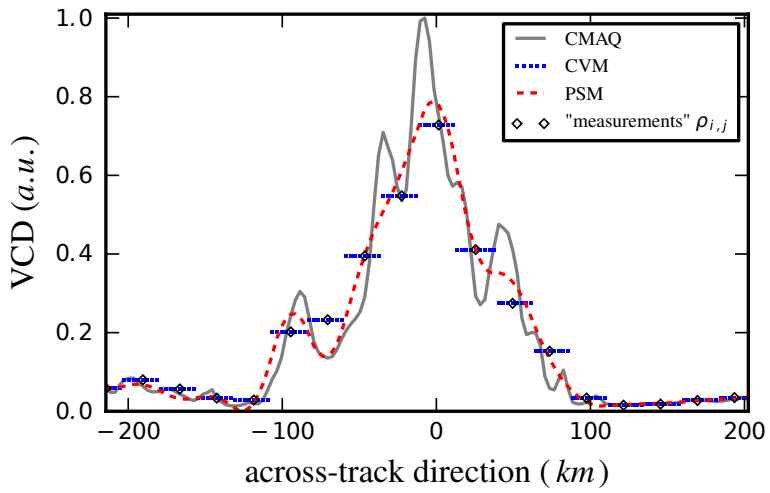

Fig. 10. Cross section through the maximum value of the CMAQ distribution in Fig. 9a and the corresponding reconstructions from Fig. 9b (CVM) and c (PSM).

$l_{\max }$ have minimum values at similar $\gamma$ independent of the measurement uncertainty $\delta_{\varepsilon}$ (Fig. 8). This suggests that the smoothing parameter $\gamma$ can be selected independently of the measurement uncertainty $\delta_{\varepsilon}$.

For one measurement $(k=1)$ the performance curves shown in Fig. 8 have a distinct minimum. Larger values for $\gamma$ increase the influence of the penalty term and lead to solutions which are smoothed excessively such that $l_{2}$ increases (smoothing error). Using a smaller $\gamma$ increases the sensitivity to measurement uncertainty $\delta_{\varepsilon}$, which also causes a larger $l_{2}$ error (perturbation error). At the swath edge, where the FWHM of the instrument function is large, the $l_{2}$ error increases strongly for small $\gamma$. As discussed in Sect. 2.4.2, solutions with $\gamma$ equal zero at the swath edge are not usable as they show severe over- and undershooting. Assessing the performance by $l_{\max }$ the curve has a minimum at a smaller $\gamma$ because $l_{\max }$ measures only the reduction of the maximum value, which is affected stronger by the smoothing error. Figure 8 shows that averaging $k$ measurement decreases the perturbation error (for small $\gamma$ ) with the inverse square root of $k$. The systematic error of the penalty term (for large $\gamma$ ) is not reduced by averaging. As a result, the minimum of the $l_{2}$ and $l_{\max }$ errors for averaged measurements is at a smaller $\gamma$ than for a single measurement. Using a wider plume, smoothing errors are smaller, and as a result it is possible to choose larger smoothing parameters (not shown).

Since our main interest is the reconstruction of distributions in source regions, $\gamma$ is chosen such that the plume is reconstructed best for small and moderate errors. Furthermore, we want avoid systematic errors resulting from large $\gamma$. Therefore, $\gamma$ is chosen such that it is slightly smaller than the minimum of $l_{2}$ for a single reconstruction. Since the position of the optimal $\gamma$ depends on the pixel overlap and thus on the FWHM of the instrument function, this optimal $\gamma$ will be different for other values of FWHM. Here, two effects are important: (1) a greater overlap increases the sensitivity to measurement uncertainties, which requires more smoothing, and (2) the same width of the plume appears narrower if the FWHM of the instrument function is larger. A wider plume has a larger optimal $\gamma$. Monte Carlo simulations are used for a fixed width of the plume $(\sigma=1 h)$ to determine the optimal $\gamma$ for varying FWHM. The optimal value for $\gamma$ decreases with increasing FWHM (not shown). This suggests that the second effect is dominant. However, it is more meaningful to measure the performance when the width of the plume is directly proportional to the FWHM, since this determines the reconstructability of a distribution. Using this scenario, the optimal smoothing parameter $\gamma$ increases with the FWHM of the instrument function. Figure 8 is used to pick the optimal $\gamma$ at nadir $(\sigma=1 h$ and FWHM about $1 h)$ and at the swath edge ( $\sigma=2 h$ and FWHM about $2 h$ ) as to be 1 and 10, respectively. The smoothing parameters for other FWHM are computed by linear interpolation between the optimal $\gamma$ at nadir and the swath edge.

Figure $6 \mathrm{~b}-\mathrm{d}$ illustrate the reconstruction of the plume in along-track direction using optimal smoothing parameters for which the distributions are reconstructed well. In addition, the reconstruction without regularisation $(\gamma=0)$ is shown, where over- and undershooting is indiscernible at 
nadir, weak near the edge and clearly visible at the swath edge (Fig. 6b-d). Hence the regularisation is mainly important for the last three rows on both edges of the swath. However, the penalty term is also suitable for reducing "fitting to noise" for all rows, as is shown next. The dependency of $l_{2}$ and $l_{\max }$ on the measurement uncertainty $\delta_{\varepsilon}$ at nadir and at the swath edge is shown in Fig. $7 \mathrm{~b}$ and c, respectively. $l_{2}$ and $l_{\max }$ errors are similar to the errors in across-track direction (Fig. 7a). The performance of PSM is improved by the penalty term, which reduces perturbation error, especially for large measurement errors $\left(\delta_{\varepsilon}>25 \%\right)$. On the other hand, the additional error due to the penalty term (smoothing error) can reduce the performance of PSM. After $k=100$ averages, because of the systematic error of the penalty term, the total error of the reconstructed plume is not a tenth of the error of a single reconstruction. However, PSM still performs similarly or better than CVM in all test scenarios.

To summarise, a suitable smoothing parameter $\gamma$ can be found empirically by considering different test scenarios. The weight of the penalty term is proportional to the absolute measurement uncertainty $\delta_{\varepsilon}$. The smoothing parameter $\gamma$ increases with the width of instrument function. The overall performance of PSM is limited by the parametrisation error and the smoothing error, which are both systematic errors. On the other hand, the penalty term is necessary to reduce the perturbation error, especially at the edge of the swath, where the FWHM of the instrument function is large. The performances of PSM and CVM are compared for different test scenarios. PSM performs similarly to CVM for large errors $\left(\delta_{\varepsilon} \geq 25 \%\right)$ and better than CVM for small and moderate errors $\left(\delta_{\varepsilon}<25 \%\right)$.

\subsubsection{The surface spline: performance and propagation of measurement uncertainty}

The algorithm described in Sect. 2.4.4 is used to reconstruct the surface spline $f_{u, v}$ for the test scenarios. The results of the Monte Carlo simulation suggest a value for the optimal smoothing parameter $\gamma$ about 1 at nadir and 10 at the edge of the swath. These values are the same as in along-track direction. Figure $7 \mathrm{~d}$ and e show the dependency of $l_{2}$ and $l_{\text {max }}$ on the measurement uncertainty $\delta_{\varepsilon}$ for the test scenarios. The results are similar to the findings for the one-dimensional spline in along-track direction.

The results can also be used to the estimate the measurement uncertainty of the trace gas map. For CVM, grid cell values are given by averaging overlapping pixels and thus the propagation error can be computed easily (Wenig et al., 2008). For PSM the computation of the error is more complex and out of the scope of this study. However, if the propagation error is dominant $\left(\delta_{\varepsilon} \geq 25 \%\right)$, Fig. 6 shows that $l_{2}$ errors for CVM and PSM are similar. Therefore, the error distribution obtained by CVM can also be used to estimate the errors of trace gas maps created by PSM.
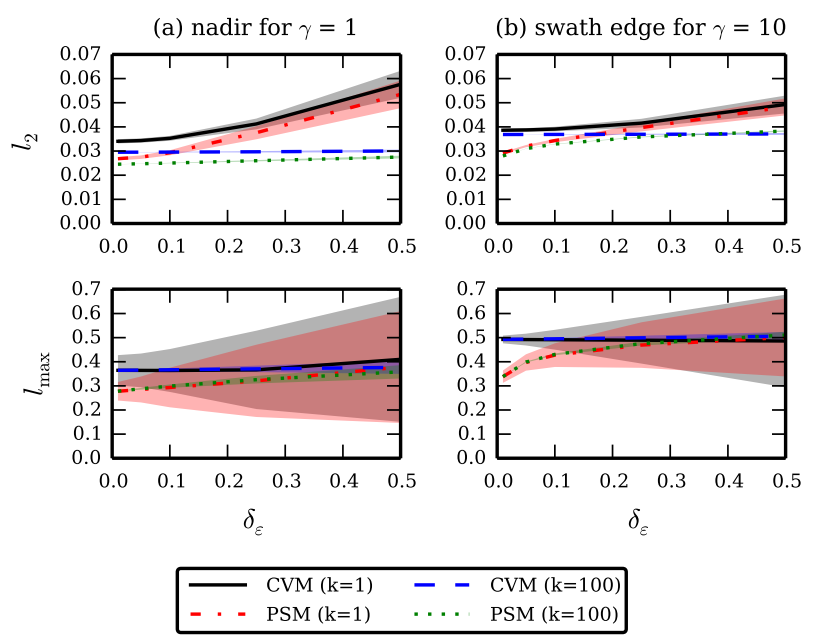

Fig. 11. Error measures for the CMAQ example in Fig. 9 for different measurement uncertainties $\delta_{\varepsilon}$. Lines indicate sample averages and the shaded areas are the standard deviations.

\subsection{Reconstruction of model distribution}

The performance of CVM and PSM is further studied using simulated $\mathrm{NO}_{2}$ distributions. We show that the above conclusions also apply for complex distributions. The simulation is performed using the Models-3 Community Multiscale Air Quality (CMAQ) Modeling System, version 4.7.1, developed by the United States Environmental Protection Agency (USEPA) (Byun and Schere, 2006). The model domain encloses the Pearl River delta region with a horizontal grid resolution of $3 \mathrm{~km}$. The meteorological input is provided by the Weather Research and Forecasting (WRF) modelling system. The air pollution emissions are based on an emissions inventory of the year 2006. The same inventory has been used in some earlier studies. The results of the simulations agree well with the observations (Huang et al., 2005; Lam et al., 2005). Figure 9 a shows the modelled $\mathrm{NO}_{2}$ column densities on 8 October 2012, 14:00 LT (local time). The distribution is normalised by its maximum value.

This model distribution is sampled using instrument functions of OMI at nadir and at the swath edge. The pixel size is assumed to be $13 \times 24 \mathrm{~km}^{2}$ in along- and across-track direction, respectively. At the swath edge, OMI pixels are actually $160 \mathrm{~km}$ long in across-track direction. However, with this coarse resolution the reconstruction of the modelled plumes would cause large parametrisation errors. For this reason, we choose a smaller pixel size to better investigate the impact of the instrument function. Again, a Monte Carlo simulation is performed to study the performance of CVM and PSM for different measurement uncertainties $\delta_{\varepsilon}$, smoothing parameters $\gamma$ and number of measurements $k$. The optimal smoothing parameters $\gamma$ are identified as 1 and 10 at nadir and at the swath edge, respectively, which are the 
(a) CVM
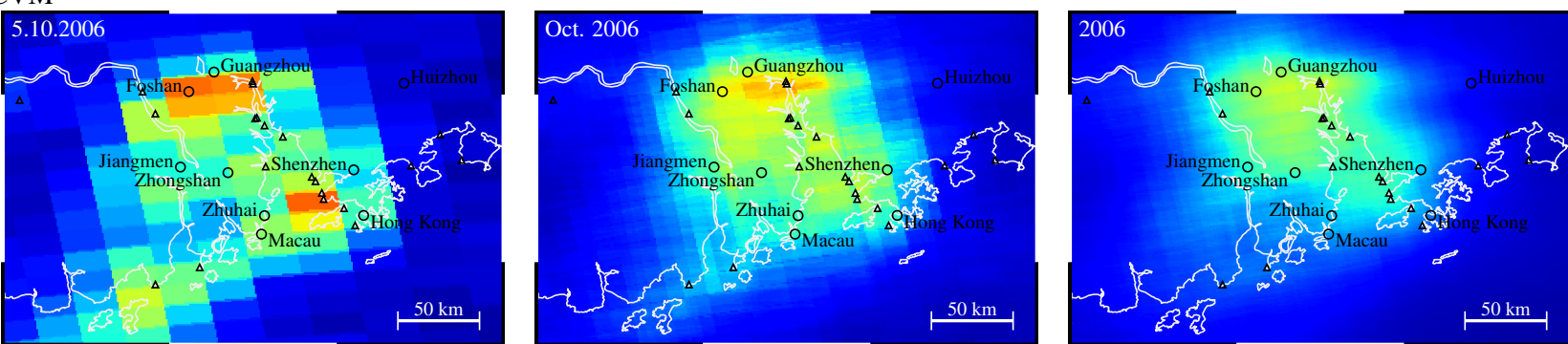

(b) PSM
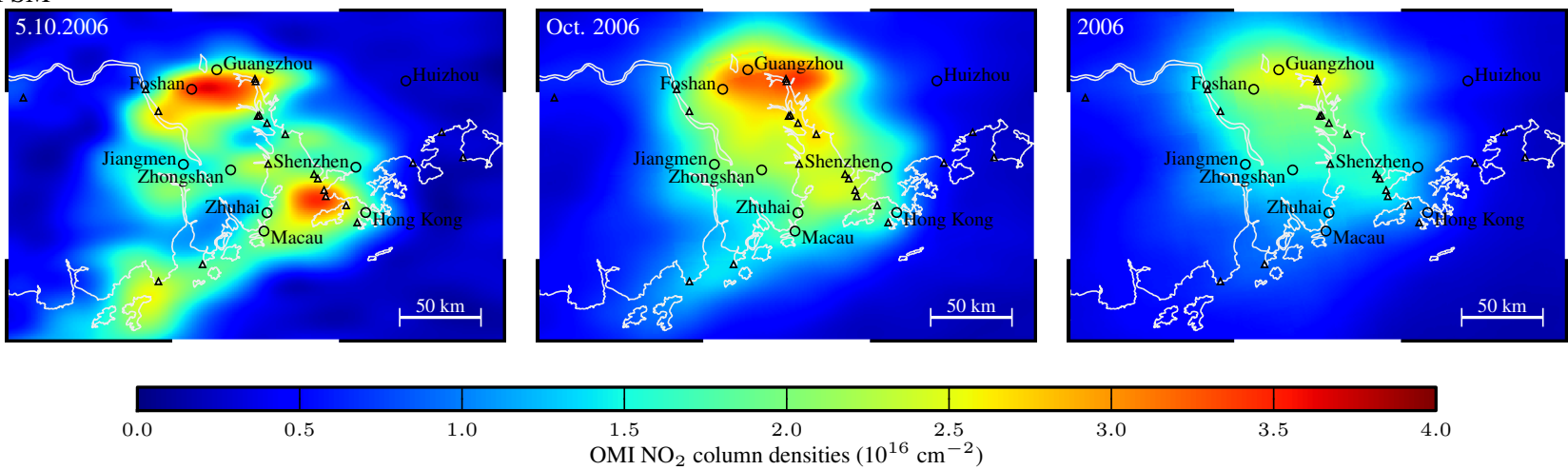

Fig. 12. Daily, monthly and annual map of tropospheric $\mathrm{NO}_{2}$ column densities over the Pearl River delta (southern China) on a $0.01^{\circ} \times 0.01^{\circ}$ grid created by (a) the standard method (CVM) and (b) our new algorithm (PSM). Locations of fossil-fuel power stations are marked as triangles.

same as in the two-dimensional test scenarios. In Fig. 11 the $l_{2}$ and $l_{\max }$ errors are shown for CVM and PSM using different measurement uncertainties $\delta_{\varepsilon}$. The results are similar to the findings obtained from the test scenarios. Figure 9 shows the reconstruction from a single measurement using a small error $\left(\delta_{\varepsilon}=0.05\right)$ for CVM and PSM. The structure of the plume - that is, the outflow towards the southwest is clearly recognisable using PSM. A cross section through the plume is given in Fig. 10. The reconstruction of the maximum value using PSM is better than for CVM. We conclude that PSM can reconstruct complex distribution over source regions with a better performance than CVM.

\section{Application to OMI data}

In this section, the new gridding algorithm (PSM) is used to create daily, monthly and annual maps of tropospheric $\mathrm{NO}_{2}$ column densities from NASA's standard product, version 2 (SP2) (Bucsela et al., 2006, 2013). Starting from 2007, OMI is affected by a row anomaly and thus several rows of each orbit are masked at the moment. To discuss the performance without the influence of the row anomaly, our analysis concentrates on measurements before 2007 .

The smoothing parameter $\gamma$ is set to 1 at nadir and 10 at the edge of the swath. In between, parameters are obtained by linear interpolation as a function of the FWHM. The scaling factor $\rho_{\text {est }}$ is set to $10^{16} \mathrm{~cm}^{-2}$, which is a typical size for high $\mathrm{NO}_{2}$ column densities. The entries of the measurement matrix $\left(\kappa^{p}\right.$ and $\left.\kappa^{d}\right)$ are pre-computed for satellite-to-ground distances between 700 and $1700 \mathrm{~km}$ and an exposure time of $2 \mathrm{~s}$ using an averaged along-track pixel size of $13.5 \mathrm{~km}$. Measurement uncertainties, provided by SP2, scale with $\mathrm{NO}_{2}$ column densities due to the air mass factor dependency of the error (Wenig et al., 2008; Bucsela et al., 2013). Using these uncertainties gives lower weights to smaller densities and hence biases distributions towards low values. To avoid this bias we adopt empirically estimated measurement uncertainties $\delta_{\varepsilon}$ based on radiative cloud fractions (RCF) (Wenig et al., 2008):

$\delta_{\varepsilon}=0.15 \times 10^{16}(1.0+3.0 \mathrm{RCF}) \mathrm{cm}^{-2} ;$

where multiple orbits overlap, column densities at a grid cell $f_{u, v}$ are the weighted average of the values in all orbits. All grid cells within the boundaries of an OMI pixel are weighted by the inverse pixel area $A_{i, j}$. This weighting function is suitable for preserving the continuity of column densities in the averaged products. In addition, the area dependency guarantees that individual grid cell errors are consistent with the error for the total pixel (Wenig et al., 2008). Using this weighting scheme, smaller OMI pixels are weighted higher, which enhances details on the map. 
(a) CVM
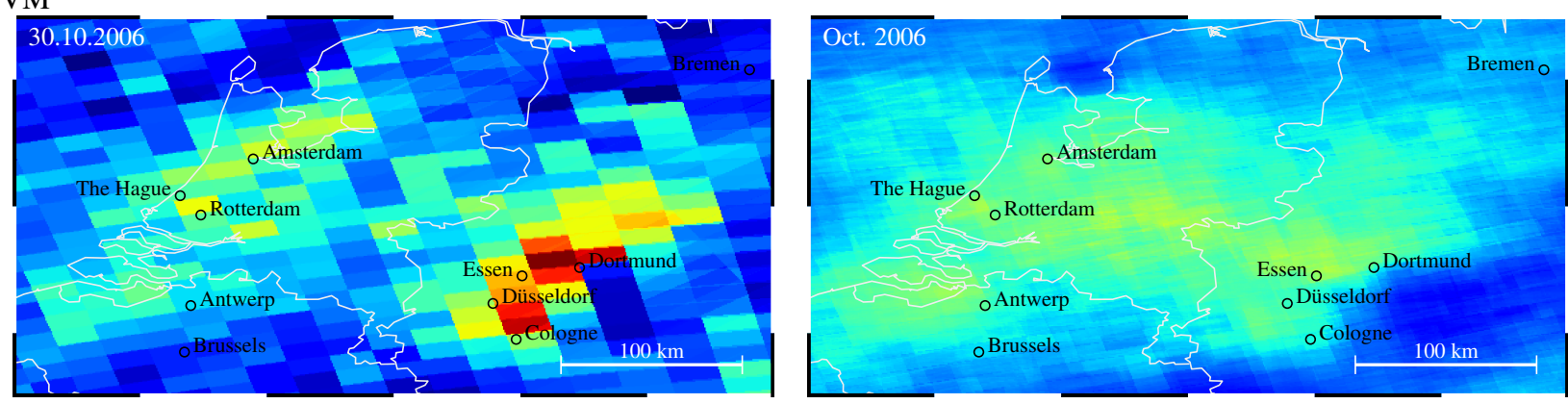

(b) PSM
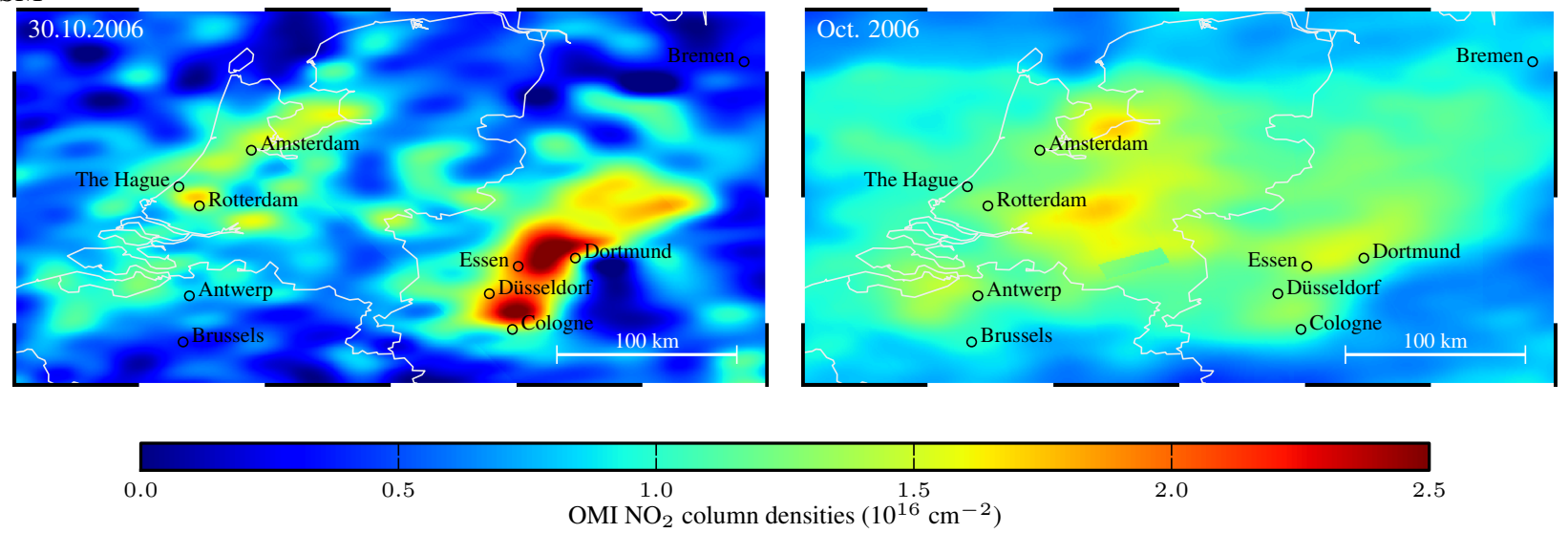

Fig. 13. Daily and monthly map of tropospheric $\mathrm{NO}_{2}$ column densities over Belgium, Germany and the Netherlands on a $0.01^{\circ} \times 0.01^{\circ}$ grid created by (a) the standard method (CVM) and (b) our new algorithm (PSM).

Daily, monthly and annual maps of tropospheric $\mathrm{NO}_{2}$ column densities are plotted for the Pearl River delta region in southern China (Fig. 12). Locations of major cities and fossil-fuel power stations are marked. The daily map created by PSM shows several distinct plumes, which have larger maximum values than the plumes in the maps produced with $\mathrm{CVM}$. The higher maximum values are likely to be closer to the true maxima. This was demonstrated in the performance evaluation in Sect. 3. The monthly map for October 2006 produced with CVM is coarse and values of adjacent grid cells may differ substantially. This spatial noisiness of the distribution makes it difficult to use column amounts at a certain location, for instance for validation studies. To obtain a reasonable value, grid cells are often averaged within a certain radius around the location. This additional step is not necessary if the maps are created by PSM as the gridding algorithm already produces a continuous distribution. As a final example, Figs. 13 and 14 show a daily and monthly distribution of $\mathrm{NO}_{2}$ column densities over Europe and the eastern USA.

\section{Discussion and conclusions}

In this study, the parabolic spline method (PSM) was developed in order to improve the accuracy and spatial resolution of trace gas maps obtained from satellite measurements. The new gridding algorithm reconstructs the trace gas distribution via a continuous parabolic spline surface using measurements from a single orbit. The spline coefficients are computed using one-dimensional splines, which allow for fast computation for a large number of pixels. PSM is the first gridding algorithm that explicitly treats the spatial sensitivity distribution of the instrument over ground using an instrument function. If the FWHM of the instrument function is about twice the length of the tiled pixel, the inverse problem is very sensitive to measurement errors. Therefore, a penalty term is used to regularise the inversion by a second-order difference matrix. This regularisation is similar to the penalty term in a smoothing spline and hence is also used to smooth measurement noise (de Boor, 2001). Monte Carlo simulations are conducted to study the performance of the algorithm for different distributions of trace gas column densities. The optimal weight of the penalty term is found to be proportional to the measurement uncertainty and the width of the instrument function.

The algorithm calculates the unknown mean values over the tiled pixel product from the measurements. While we use these values as spline coefficients, it should be noted that they can also be used directly as a better approximation 
(a) CVM
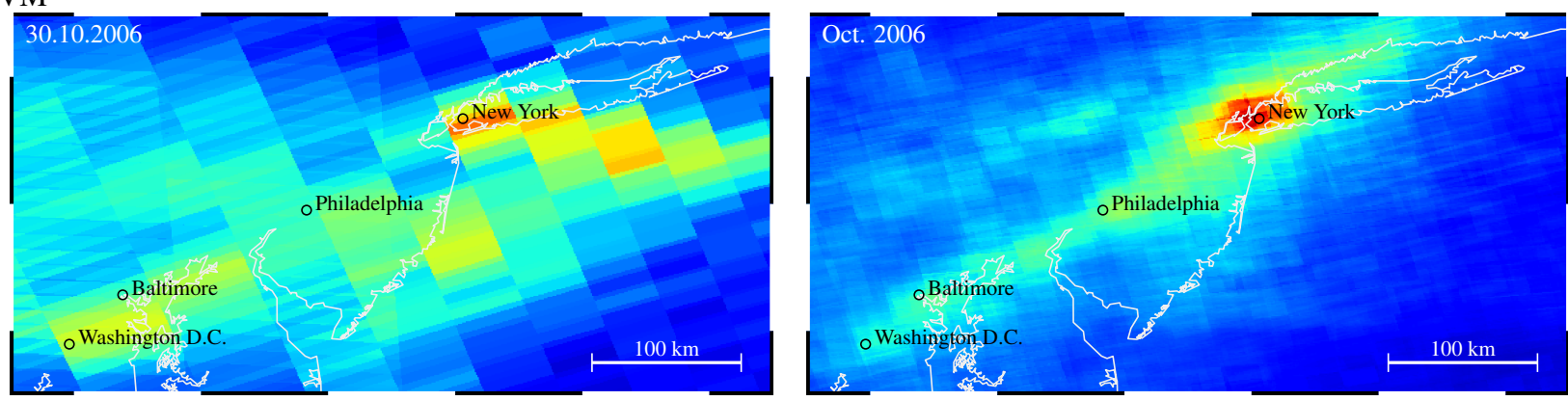

(b) PSM
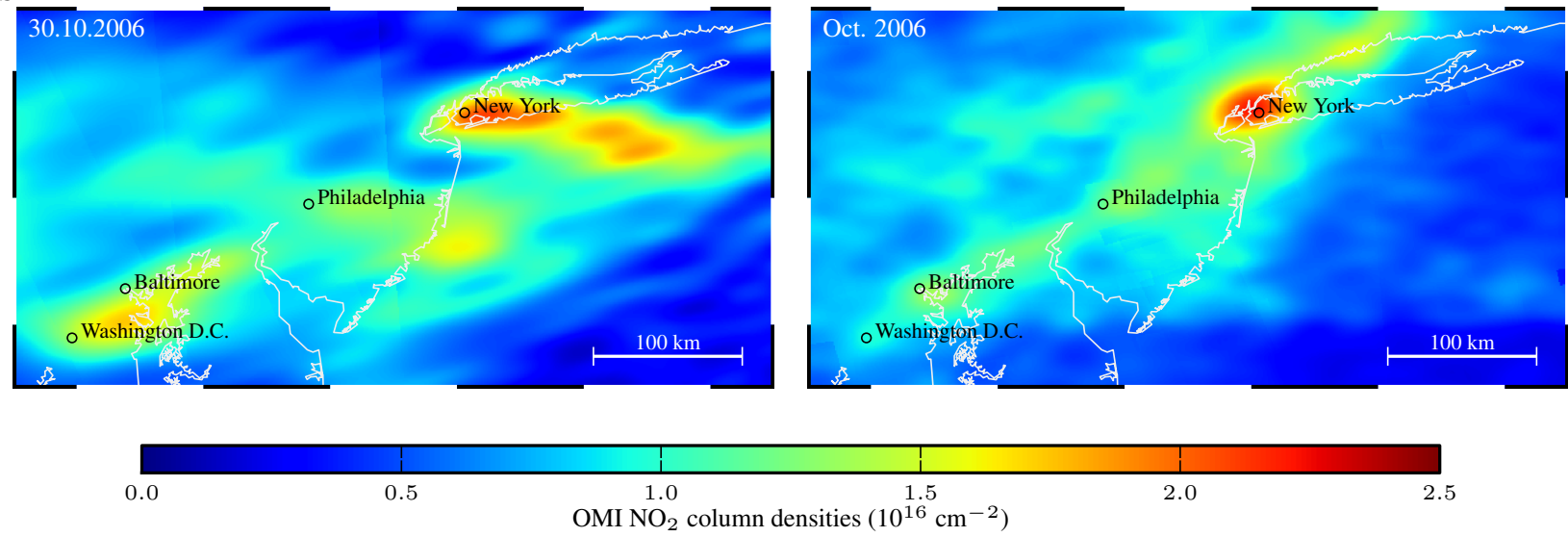

Fig. 14. Daily and monthly map of tropospheric $\mathrm{NO}_{2}$ column densities over the eastern USA on a $0.01^{\circ} \times 0.01^{\circ}$ grid created by (a) the standard method (CVM) and (b) our new algorithm (PSM).

to visualise the mean values over the tiled pixels. This approach is more accurate than the often-used assumption that the mean values over the tiled pixel are equivalent to the measurement values. A related study on the best approximation of mean values over tiled pixels is planned in the future.

The reason for the sensitivity of the inverse problem to measurement noise has not been analysed in detail in this paper. Our analysis suggests that the inverse problem becomes undetermined because the measurement noise and the wide instrument function reduce the "effective" rank of the problem. In addition, the parametrisation of the distribution by parabolic splines may favour the oscillation of the mean values because the spline is created by combining parabolas with continuous first derivatives. In this study, we use parabolic splines because they are the simplest parametrisation. A different parametrisation, for example higher-order polynomials, could make the inversion more stable.

The performance of PSM is compared with a standard algorithm, described by Wenig et al. (2008), which assumes constant measurement values within pixel boundaries and thus is named the constant value method (CVM) here. Due to the smaller parametrisation errors, reconstructions using PSM are better than CVM if the perturbation error is small or is reduced by averaging. Trace gas maps created by PSM are smoother and maximum values are better reconstructed. Better performance is also illustrated by reconstructing the high-resolution distribution calculated with the CMAQ chemistry model. Application to $\mathrm{OMI} \mathrm{NO}_{2}$ column densities suggests that PSM is more suitable than CVM for creation of high-resolution maps. It should be understood that the best possible resolution is fundamentally limited by the spatial resolution of the satellite instrument. However, a well-chosen gridding algorithm may be able to detect smallscale features at this limit, which are missed otherwise. The use of PSM in future satellite validation studies is being planned.

The general approach described in this study can be easily extended for higher-order splines, for instance quartic splines, to further improve the parametrisation of the distribution. The gridding algorithm can also be applied to other OMI-like instruments such as the TROPOspheric Monitoring Instrument (TROPOMI), planned for launch in 2014 (Veefkind et al., 2012). The high spatial resolution of TROPOMI $\left(7 \times 7 \mathrm{~km}^{2}\right.$ at nadir) allows for the detection of small-scale features and is likely to reduce the measurement uncertainty due to more cloud-free observations. 
In this paper, we demonstrate that the newly developed gridding algorithm improves the reconstruction of regional trace gas maps. Its application could be very helpful when satellite-derived trace gas distributions are studied on a regional scale.

Acknowledgements. The work described in this paper is partly funded by the Guy Carpenter Asia-Pacific Climate Impact Centre (project no. 9360126) and a grant from the Research Grant Council of Hong Kong (project no. 102912).

Edited by: D. Loyola

\section{References}

Beirle, S., Platt, U., Wenig, M., and Wagner, T.: Weekly cycle of $\mathrm{NO}_{2}$ by GOME measurements: a signature of anthropogenic sources, Atmos. Chem. Phys., 3, 2225-2232, doi:10.5194/acp3-2225-2003, 2003.

Boersma, K. F., Eskes, H. J., Veefkind, J. P., Brinksma, E. J., van der A, R. J., Sneep, M., van den Oord, G. H. J., Levelt, P. F., Stammes, P., Gleason, J. F., and Bucsela, E. J.: Near-real time retrieval of tropospheric $\mathrm{NO}_{2}$ from OMI, Atmos. Chem. Phys., 7, 2103-2118, doi:10.5194/acp-7-2103-2007, 2007.

Boersma, K. F., Eskes, H. J., Dirksen, R. J., van der A, R. J., Veefkind, J. P., Stammes, P., Huijnen, V., Kleipool, Q. L., Sneep, M., Claas, J., Leitão, J., Richter, A., Zhou, Y., and Brunner, D.: An improved tropospheric $\mathrm{NO}_{2}$ column retrieval algorithm for the Ozone Monitoring Instrument, Atmos. Meas. Tech., 4, 1905-1928, doi:10.5194/amt-4-1905-2011, 2011.

Brunner, D., Schaub, D., and Buchmann, B.: Reconstructing finescale air pollution structures from coarsely resolved satellite observations, in: Proceedings of Envisat Symposium 2007, Montreux, Switzerland, ESA Communication Production Office and ESTEC, Noordwijk, the Netherlands, 2007.

Bucsela, E., Celarier, E., Wenig, M., Gleason, J., Veefkind, J., Boersma, K., and Brinksma, E.: Algorithm for $\mathrm{NO}_{2}$ vertical column retrieval from the Ozone Monitoring Instrument, IEEE T. Geosci. Remote, 44, 1245-1258, doi:10.1109/TGRS.2005.863715, 2006.

Bucsela, E. J., Krotkov, N. A., Celarier, E. A., Lamsal, L. N., Swartz, W. H., Bhartia, P. K., Boersma, K. F., Veefkind, J. P., Gleason, J. F., and Pickering, K. E.: A new stratospheric and tropospheric $\mathrm{NO}_{2}$ retrieval algorithm for nadir-viewing satellite instruments: applications to OMI, Atmos. Meas. Tech., 6, 26072626, doi:10.5194/amt-6-2607-2013, 2013.

Byun, D. and Schere, K. L.: Review of the governing equations, computational algorithms, and other components of the Models3 Community Multiscale Air Quality (CMAQ) modeling system, Appl. Mech. Rev., 59, 51-57, doi:10.1115/1.2128636, 2006.

Carn, S. A., Krueger, A. J., Krotkov, N. A., Yang, K., and Levelt, P. F.: Sulfur dioxide emissions from Peruvian copper smelters detected by the Ozone Monitoring Instrument, Geophys. Res. Lett., 34, L09801, doi:10.1029/2006GL029020, 2007.

Chan, K. L., Pöhler, D., Kuhlmann, G., Hartl, A., Platt, U., and Wenig, M. O.: $\mathrm{NO}_{2}$ measurements in Hong Kong using LED based long path differential optical absorption spectroscopy, At- mos. Meas. Tech., 5, 901-912, doi:10.5194/amt-5-901-2012, 2012.

Choi, Y., Wang, Y., Zeng, T., Cunnold, D., Yang, E.-S., Martin, R., Chance, K., Thouret, V., and Edgerton, E.: Springtime transitions of $\mathrm{NO}_{2}, \mathrm{CO}$, and $\mathrm{O}_{3}$ over North America: model evaluation and analysis, J. Geophys. Res., 113, D20311, doi:10.1029/2007JD009632, 2008.

de Boor, C.: A practical guide to splines, Springer Verlag, New York, Berlin, Heidelberg, 2001.

Dirksen, R. J., Boersma, K. F., Eskes, H. J., Ionov, D. V., Bucsela, E. J., Levelt, P. F., and Kelder, H. M.: Evaluation of stratospheric $\mathrm{NO}_{2}$ retrieved from the Ozone Monitoring Instrument: intercomparison, diurnal cycle, and trending, J. Geophys. Res.Atmos., 116, D08305, doi:10.1029/2010JD014943, 2011.

Huang, J.-P., Fung, J. C., Lau, A. K., and Qin, Y.: Numerical simulation and process analysis of typhoon-related ozone episodes in Hong Kong, J. Geophys. Res.-Atmos., 110, D05301, doi:10.1029/2004JD004914, 2005.

Kobza, J. and Mlcak, J.: Biquadratic splines interpolating mean values, Appl. Math-Czech, 39, 339-356, 1994.

Krotkov, N. A., McClure, B., Dickerson, R. R., Carn, S. A., Li, C., Bhartia, P. K., Yang, K., Krueger, A. J., Li, Z., Levelt, P. F., Chen, H., Wang, P., and Lu, D.: Validation of $\mathrm{SO}_{2}$ retrievals from the Ozone Monitoring Instrument over NE China, J. Geophys. Res., 113, D16S40, doi:10.1029/2007JD008818, 2008.

Kurosu, T. P. and Celarier, E. A.: OMIPIXCOR Readme File, available at: http://disc.sci.gsfc.nasa.gov/Aura/data-holdings/OMI/ documents/v003/OMPIXCOR_README_V003.pdf (last access: August 2013), 2010.

Lam, K., Wang, T., Wu, C., and Li, Y.: Study on an ozone episode in hot season in Hong Kong and transboundary air pollution over Pearl River Delta region of China, Atmos. Environ., 39, 19671977, doi:10.1016/j.atmosenv.2004.11.023, 2005.

Lancaster, P. and Salkauskas, K.: Curve and Surface Fitting - An Introduction, Academic Press Ltd., London, 1986.

Levelt, P., van den Oord, G., Dobber, M., Malkki, A., Visser, H., de Vries, J., Stammes, P., Lundell, J., and Saari, H.: The Ozone Monitoring Instrument, IEEE T. Geosci. Remote, 44, 10931101, doi:10.1109/TGRS.2006.872333, 2006.

Martin, R. V., Jacob, D. J., Chance, K., Kurosu, T. P., Palmer, P. I., and Evans, M. J.: Global inventory of nitrogen oxide emissions constrained by space-based observations of $\mathrm{NO}_{2}$ columns, J. Geophys. Res., 108, 4537, doi:10.1029/2003JD003453, 2003.

Nocedal, J. and Wright, S. J.: Numerical Optimization, Springer Science + Business Media, New York, 2006.

Richter, A., Burrows, J. P., Nüß, H., Granier, C., and Niemeier, U.: Increase in tropospheric nitrogen dioxide over China observed from space, Nature, 437, 129-132, doi:10.1038/nature04092, 2005.

Russell, A. R., Perring, A. E., Valin, L. C., Bucsela, E. J., Browne, E. C., Wooldridge, P. J., and Cohen, R. C.: A high spatial resolution retrieval of $\mathrm{NO}_{2}$ column densities from OMI: method and evaluation, Atmos. Chem. Phys., 11, 8543-8554, doi:10.5194/acp-11-8543-2011, 2011.

Späth, H.: One Dimensional Spline Interpolation Algorithms, AK Peters, Wellesley, MA, USA, 1995.

Veefkind, J., Aben, I., McMullan, K., Förster, H., de Vries, J., Otter, G., Claas, J., Eskes, H., de Haan, J., Kleipool, Q., van Weele, M., Hasekamp, O., Hoogeveen, R., Landgraf, J., 
Snel, R., Tol, P., Ingmann, P., Voors, R., Kruizinga, B., Vink, R., Visser, H., and Levelt, P.: TROPOMI on the ESA Sentinel-5 precursor: a GMES mission for global observations of the atmospheric composition for climate, air quality and ozone layer applications, Remote Sens. Environ., 120, 70-83, doi:10.1016/j.rse.2011.09.027, 2012.

Wenig, M. O., Cede, A. M., Bucsela, E. J., Celarier, E. A., Boersma, K. F., Veefkind, J. P., Brinksma, E. J., Gleason, J. F., and Herman, J. R.: Validation of OMI tropospheric $\mathrm{NO}_{2}$ column densities using direct-Sun mode Brewer measurements at NASA Goddard Space Flight Center, J. Geophys. Res., 113, D16S45, doi:10.1029/2007JD008988, 2008.
Zhou, Y., Brunner, D., Boersma, K. F., Dirksen, R., and Wang, P.: An improved tropospheric $\mathrm{NO}_{2}$ retrieval for OMI observations in the vicinity of mountainous terrain, Atmos. Meas. Tech., 2, 401-416, doi:10.5194/amt-2-401-2009, 2009.

Zhou, Y., Brunner, D., Spurr, R. J. D., Boersma, K. F., Sneep, M., Popp, C., and Buchmann, B.: Accounting for surface reflectance anisotropy in satellite retrievals of tropospheric $\mathrm{NO}_{2}$, Atmos. Meas. Tech., 3, 1185-1203, doi:10.5194/amt-3-11852010, 2010. 